SLAC-PUB-8245

September 1999

hep-th/9909072

EFI-99-35

YCTP-22-99

SU-ITP-99/42

\title{
Tension is Dimension
}

Jeffrey A. Harvey et al.

Submitted to Journal of High Energy Physics (JHEP)

Stanford Linear Accelerator Center, Stanford University, Stanford, CA 94309

Work supported by Department of Energy contract DE-AC03-76SF00515. 
hep-th/9909072

EFI-99-35

YCTP-22-99

SU-ITP-99/42

SLAC-PUB- 8245

\title{
Tension is Dimension
}

\author{
Jeffrey A. Harvey*, Shamit Kachru», Gregory Moore ${ }^{\star 1 i}$ and Eva Silverstein ${ }^{\wedge}$ \\ - Enrico Fermi Institute and Department of Physics \\ University of Chicago, 5640 Ellis Avenue, Chicago, IL 60637 \\ $\diamond$ Department of Physics and SLAC \\ Stanford University \\ Stanford, CA 94305/94309 \\ - Department of Physics, Yale University, \\ Box 208120, New Haven, CT 06520
}

\begin{abstract}
We propose a simple universal formula for the tension of a D-brane in terms of a regularized dimension of the associated conformal field theory statespace.
\end{abstract}

September 10, 1999

1 Address after Jan. 1, 2000: Dept. of Physics and Astronomy, Rutgers University, Piscataway, NJ 08855-0849 


\section{Introduction}

D-branes are destined to play a fundamental role in the formulation of nonperturbative string theory. Nevertheless, despite much work, and a good understanding of examples such as toroidal compactification, there is as yet no general formulation of the D-brane spectrum applicable to an arbitrary closed string background. In this note we point out one simple aspect of D-branes which, we conjecture, is quite general. Namely, the square of the tension of a D-brane is proportional to the regularized dimension of some infinite dimensional algebra. We show that this is indeed true for the wide class of D-branes associated to rational and quasi-rational conformal field theories.

While the general construction of the D-brane spectrum has yet to be carried out, much is indeed known thanks to recent vigorous development of boundary conformal field theory, and the boundary state formalism. A partial list of references includes 传, states $|B\rangle\rangle$ are linear functionals on the closed string statespace $\mathcal{H}^{\text {closed }}$ preserving conformal invariance

$$
\left.\left(L_{n}-\tilde{L}_{-n}\right)|B\rangle\right\rangle=0
$$

where $L_{n}, \tilde{L}_{n}$ are the left and right Virasoro generators.

One might think that (i1: $\left.\overline{1}_{1}^{\prime}\right)$, which imposes conformal invariance on the string worldsheet, is the only condition one needs to impose to find physically acceptable boundary states in string theory. Taking this point of view immediately leads to problems: there

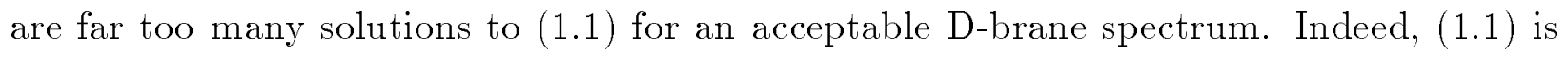
a linear equation and solutions are in one to one correspondence with spinless Virasoro primaries $\phi_{h, \tilde{h}}^{\alpha}$ in the closed string spectrum [i-1.

This result is most naturally understood as follows [0. We can decompose $\mathcal{H}^{\text {closed }}$ in terms of Virasoro irreps $V_{h}$ as

$$
\mathcal{H}^{\text {closed }}=\oplus_{(h, \tilde{h}) \in \operatorname{Spec}} V_{h} \otimes \tilde{V}_{\tilde{h}}
$$

where the spectrum of the closed string theory is determined by the set Spec of pairs of conformal dimensions. We can then solve (1: $(1,1)$ in each component of $\mathcal{H}^{\text {closed }}$ as

$$
|B\rangle\rangle_{h, \tilde{h}}=\sum_{m, \tilde{m}} a_{m, \tilde{m}}|h, m\rangle \otimes|\tilde{h}, \tilde{m}\rangle \in V_{h} \otimes \tilde{V}_{\tilde{h}} .
$$


Using the inner product on $\tilde{V}_{\tilde{h}}$ under which $\left.L_{n}^{\dagger}=L_{-n},|B\rangle\right\rangle_{h, \tilde{h}}$ is equivalent to a homomorphism $B_{h, \tilde{h}}: \tilde{V}_{\tilde{h}} \rightarrow V_{h}$ given by

$$
B_{h, \tilde{h}}=\sum_{m, \tilde{m}} a_{m, \tilde{m}}|h, m\rangle \otimes\langle\tilde{h}, \tilde{m}|
$$

obeying $L_{n} B_{h, \tilde{h}}=B_{h, \tilde{h}} \tilde{L}_{n}$. That is, $B_{h, \tilde{h}}$ is an intertwiner between $V_{h}$ and $\tilde{V}_{\tilde{h}}$. Since these are irreps it follows from Schur's lemma that that $|B\rangle\rangle_{h, \tilde{h}}$ vanishes if $h \neq \tilde{h}$ and that $B_{h, \tilde{h}}$ is proportional to the identity when $h=\tilde{h}$ (the fact that we need $h=\tilde{h}$ is already clear from the $n=0$ component of $\left.\left(i_{2}^{-} \cdot \overline{1}_{1}^{1}\right)\right)$. Choosing the proportionality constant to be one, we can take $B$ acting on $\mathcal{H}^{\text {closed }}$ to be the projection operator onto $V_{h} \otimes \tilde{V}_{\tilde{h}=h}$. We denote the corresponding "states" in $\mathcal{H}^{\text {closed }}$ by $\left.|h, \tilde{h}=h\rangle\right\rangle$. There is a natural generalization of this construction to arbitrary chiral algebras [1] referred to as "Ishibashi states." We will refer to them as "character states."

One problem with these Virasoro character states is that most of them do not couple to the graviton at leading order in string perturbation theory. This follows because the overlap with the graviton state of a primary or descendent in another irrep (not containing the graviton state as primary) is zero. Therefore, if one wrapped these "branes" on cycles the resulting particles would have string scale $\mathcal{O}\left(g_{s}^{0}\right)$ masses and one would expect severe problems with unitarity.

On the other hand, it has been well appreciated for some time that in addition to

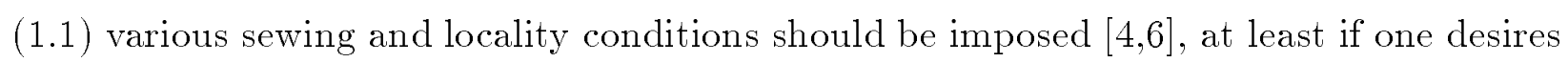
a description in terms of local boundary conformal field theory on the string worldsheet. An especially important role is played by the Cardy condition [3] . To state this condition consider possible boundary states $|\alpha\rangle\rangle,|\beta\rangle\rangle$, and compute the partition function (cylinder amplitude)

$$
Z_{\alpha \beta}=\left\langle\left\langle\beta\left|q_{c}^{\frac{1}{2}\left(L_{0}^{c}+\tilde{L}_{0}^{c}-\frac{c}{12}\right)}\right| \alpha\right\rangle\right\rangle
$$

where $L_{0}^{c}, \tilde{L}_{0}^{c}$ are the left and right-moving closed string Hamiltonians and $q_{c}=e^{-2 \pi t_{c}}$. This can be given a Hamiltonian interpretation in the open string channel by viewing the cylinder as an annulus with Euclidean time running around the annulus. After a conformal rescaling of coordinates we should thus be able to write (

$$
\operatorname{Tr}_{H_{\alpha \beta} q_{o}} L_{\mathrm{o}}^{\mathrm{open}}-c / 24
$$

where $H_{\alpha \beta}$ is the Hilbert space of open strings with boundary conditions defined by $\alpha, \beta$ and $q_{o}=e^{-2 \pi t_{o}}=e^{-2 \pi / t_{c}}$. Cardy's condition follows from the equality of ('1. 
In other words, the modular transform of (11... with non-negative integer coefficients for all possible pairs of boundary states $|\alpha\rangle\rangle,|\beta\rangle\rangle$. We will call such a set of boundary states a Cardy set.

In the following we demonstrate that imposing the Cardy condition reduces the solu-

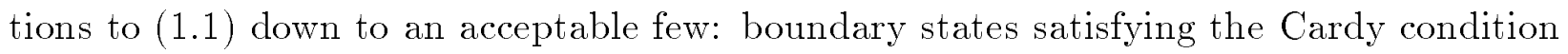
must couple to the graviton at leading order in string perturbation theory, and therefore have tensions of order $\mathcal{O}\left(g_{s}^{-1}\right)$.

Moreover, we find that the tensions are given by the (suitably regularized) dimension of an associated open string statespace as in equations (2.3) and (3.13) below. We believe that the generality of this result has not been appreciated previously, although many of the elements of our argument are not new. In particular, the regularized dimension has appeared previously in the literature on boundary CFT as the boundary entropy of Affleck

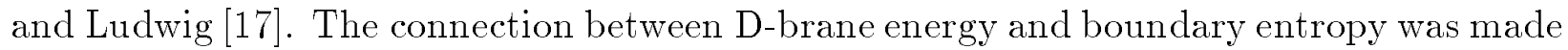
in [4] for D-branes moving in flat space. Furthermore, we discovered after completing this work that our computation of the boundary entropy for torus compactifications in sec 2.2 below appeared earlier in [i] $\overline{1} \overline{1}$.

\section{The general argument: Bosonic string}

We consider a spacetime defined by a closed conformal field theory of the type $\mathcal{C}\left(\mathbb{R}^{1,25-d}\right) \otimes \mathcal{C}_{2}$. The first factor is the usual conformal field theory of $26-d$ free uncompactified bosons and ghosts. For our purposes it suffices to work in light-cone gauge in which case we can drop the ghost fields and view the first factor as the CFT of $24-d$ free bosons. The second factor is an arbitrary unitary CFT of $c=\bar{c}=d$. We will choose $d$ sufficiently large so that we can view the $D p$-brane we are interested in as a D-particle in $26-d$ spacetime dimensions.

\subsection{D-brane Tension}

We consider D-brane boundary states of the form:

$$
|x\rangle\rangle \otimes|\alpha\rangle\rangle
$$

Here $|\alpha\rangle\rangle$ is a boundary state for $\mathcal{C}_{2}$ which is assumed to be part of a Cardy set. It corresponds to an open string channel statespace $\mathcal{H}_{\alpha \alpha}$ as in ('- $\left.{ }_{1}^{\prime}\right)$. The first factor $\left.|x\rangle\right\rangle$ is 
a standard position eigenstate D-brane state constructed from coherent states of lightcone gauge oscillators.

$$
|x\rangle\rangle:=\mathcal{N}_{\text {s.t. }} \int d^{24-d} k e^{i k x} e^{-\sum \frac{1}{n} \alpha_{-n} \tilde{\alpha}_{-n}}|k\rangle
$$

Here $|k\rangle$ denotes a momentum eigenstate in the closed string Hilbert space; the normalization $\mathcal{N}_{\text {s.t. }}$ can be gleaned from [isi] and involves powers of $2, \pi, \ell_{s}$.

The D-brane state $\left(2, \overline{1}_{1}^{\prime}\right)$ describes a particle in the $(26-d)$-dimensional spacetime theory. The formula for the mass of this particle in terms of $\mathcal{H}_{\alpha \alpha}$ is:

$$
\left(\ell_{s} M\right)^{2}=\frac{1}{(64 \pi)^{2}} \frac{\left(2 \pi \ell_{s}\right)^{24-d}}{G_{26-d}} \operatorname{dim} \mathcal{H}_{\alpha \alpha}
$$

where $G_{26-d}$ is the Newton constant in 26-d dimensions, $\ell_{s}$ is the string length $\left(\alpha^{\prime}=\ell_{s}^{2}\right)$ and $\operatorname{dim} \mathcal{H}_{\alpha \alpha}$ is the regularized dimension, defined by

$$
\operatorname{dim} \mathcal{H}_{\alpha \alpha}=\lim _{\tau \rightarrow 0} e^{2 \pi i c / 24(-1 / \tau)} \operatorname{Tr}_{\mathcal{H}_{\alpha \alpha}} q^{L_{0}-c / 24}
$$

where $q=e^{2 \pi i \tau}$.

In order to prove $\left(2 \overline{3}^{\prime}\right)$ we will assume that $\mathcal{C}_{2}$ is described by rational conformal field theory (RCFT). We believe that this is only a technical assumption and that (2. more generally. Some evidence for this will be given later where (2.2. in quasirational theories.

In RCFT we have isomorphic left and right-moving chiral algebras $\mathcal{A}_{L}=\mathcal{A}_{R}=\mathcal{A}$ which contain the Virasoro algebra and which may in general be subalgebras of a larger chiral algebra. We denote the moments of the chiral fields generating $\mathcal{A}$ by $W_{n}$. By definition, the Hilbert space can be decomposed into a finite set of irreps $V_{j}$ of $\mathcal{A}$

$$
\mathcal{H}^{\text {closed }}=\oplus_{(j, \tilde{j}) \in \operatorname{Spec}} V_{j} \otimes \tilde{V}_{j}
$$

with Spec labelling the irreps in the spectrum, possibly with multiplicities $N_{j, j}$. The characters

$$
\chi_{i}(\tau)=\operatorname{Tr}_{V_{i}} q^{L_{0}-c / 24}
$$

transform under modular transformations $\tau \rightarrow-1 / \tau$ according to

$$
\chi_{i}(-1 / \tau)=\sum_{j} S_{i}{ }^{j} \chi_{j}(\tau)
$$


For each irrep $V_{i}$ there is a primary field $\phi_{i}$ obeying the fusion algebra

$$
\phi_{i} \times \phi_{j}=N_{i j}^{k} \phi_{k}
$$

with structure constants related to the $\mathrm{S}$ matrix by the Verlinde formula

$$
N_{i j k}=\sum_{\ell} \frac{S_{i}{ }^{\ell} S_{j}{ }^{\ell} S_{k}^{\ell}}{S_{1}{ }^{\ell}}
$$

Demanding that $\mathcal{A}$ act in the RCFT with boundary requires that

$$
\left.\left(W_{n}-(-1)^{h_{W}} \Omega\left(\bar{W}_{-n}\right)\right)|\alpha\rangle\right\rangle=0
$$

where $h_{W}$ is the conformal dimension (spin) of $W$ and $\Omega$ is an automorphism of $\mathcal{A}$. Character states $|j\rangle\rangle$ solving $\left(2_{2}^{-} \overline{1}_{1}^{\prime}\right)$ can be constructed by a slight variant of the argument given earlier $\left[\begin{array}{c}1 \\ 6\end{array}\right.$ character states form a basis, we can write possible elements of a Cardy set as

$$
\left.|\alpha\rangle\rangle=\sum_{j} \frac{\psi_{\alpha}^{j}}{{\sqrt{S_{1}}}^{j}}|j\rangle\right\rangle
$$

where the factor in the denominator has been put in for later convenience. It is positive, see below.

Using

$$
\left\langle\left\langle j\left|q_{c}^{\frac{1}{2}\left(L_{0}+\tilde{L}_{0}-\frac{c}{12}\right)}\right| k\right\rangle\right\rangle=\delta_{j k} \chi_{j}\left(q_{c}\right)
$$

we then have

$$
Z_{\beta \alpha}=\sum_{j} \frac{\psi_{\alpha}^{j}\left(\psi_{\beta}^{j}\right)^{*}}{S_{1}{ }^{j}} \chi_{j}\left(q_{c}\right)=\sum_{j, k} \frac{\psi_{\alpha}^{j}\left(\psi_{\beta}^{j}\right)^{*}}{S_{1}{ }^{j}} S_{j}^{k} \chi_{k}\left(q_{o}\right)
$$

On the other hand, since $\mathcal{A}$ acts on the open string Hilbert space, we can also decompose $\mathcal{H}_{\alpha \beta}$ into $\mathcal{A}$ irreps so that

$$
\operatorname{Tr}_{H_{\alpha \beta}} q_{o}^{L_{0}-c / 24}=\sum_{i}\left(n^{i}\right)_{\alpha \beta} \chi_{i}\left(q_{o}\right)
$$

with $n_{\alpha \beta}^{i}$ non-negative integers. Equating ( $\left(2,14^{\prime}\right)$ and $\left(2,13^{i}\right)$ then gives Cardy's condition in the context of boundary RCFT:

$$
\left(n^{i}\right)_{\alpha \beta}=\sum_{j} \frac{S_{i}{ }^{j}}{S_{1}{ }^{j}} \psi_{\alpha}^{j}\left(\psi_{\beta}^{j}\right)^{*}
$$


The solution originally given by Cardy has $\alpha$ running over the irreps of $\mathcal{A}$ and $\left(n^{i}\right)_{\alpha \beta}=$

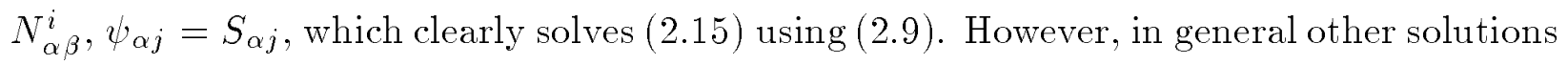

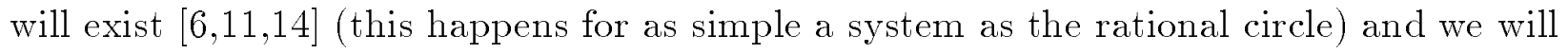
only assume (2.2.

We are now ready to prove ( $(2.3)$. The key observation is that the mass is measured by the one-point function with the graviton. The graviton vertex operator $\epsilon_{\mu \nu} \partial X^{\mu} \bar{\partial} X^{\nu} e^{i k \cdot X} \otimes$ 1 is the unit operator in the internal theory $\mathcal{C}_{2}$. Thus we need only know the coupling of $|\alpha\rangle\rangle$ to the character of the representation with the unit operator in order to compute the mass of the D-brane.

The unit operator is in a unique character state $|1\rangle\rangle$ and uniqueness of the vacuum implies $\langle 0 \mid 1\rangle\rangle=1$. Therefore, the dependence of the tension on the internal conformal field theory is exactly $\psi_{\alpha}^{1} / \sqrt{S_{11}}$. On the other hand, the regularized dimension of $\mathcal{H}_{\alpha \alpha}$ is

$$
\begin{aligned}
\operatorname{dim} \mathcal{H}_{\alpha \alpha} & =\lim _{q_{o} \rightarrow 1} q_{c}^{c / 24} \operatorname{Tr}_{\mathcal{H}_{\alpha \alpha}} q_{o}^{L_{0}-c / 24} \\
& =\lim _{q_{o} \rightarrow 1} q_{c}^{c / 24} \sum_{j, k} \frac{S_{k}{ }^{j}}{S_{1}{ }^{k}}\left|\psi_{\alpha}^{k}\right|^{2} \chi_{j}\left(q_{o}\right) \\
& =\frac{\left|\psi_{\alpha}\right|^{2}}{S_{11}}
\end{aligned}
$$

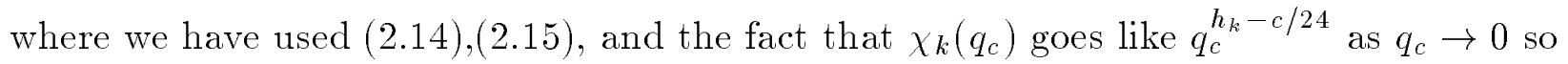
that the dominant contribution comes from the identity representation with $h_{1}=0$ in the limit. This then proves (2.3. the torus (see below) and comparing to [20-0]

It is easy to show that the dimension (and hence the tension) is nonzero, at least in RCFTs. In the Cardy condition we impose positivity $\left(n^{j}\right)_{\alpha \beta} \geq 0$ and the vacuum must appear in $\left(n^{0}\right)_{\alpha \alpha} \geq 1$. Moreover, the matrix element $S_{j 1} \geq 0$. This latter fact is easily proved since the regularized dimension of the representation $j$ of the chiral algebra is given by the modular matrix [2]1]:

$$
\operatorname{dim} \mathcal{H}_{j}=\lim _{t \rightarrow 0} \frac{\operatorname{Tr}_{\mathcal{H}_{j}} e^{-2 \pi t H}}{\operatorname{Tr}_{\mathcal{H}_{1}} e^{-2 \pi t H}}=\frac{S_{j 1}}{S_{11}}
$$

This is a limit of positive quantities and hence nonnegative. cannot vanish because that leads to inconsistencies in the modular representation. Indeed

2 We are assuming unitarity of the internal CFT $\mathcal{C}_{2}$ here, which is reasonable for string theory applications. 
the interpretation of this quantity as a positive dimension is crucial to the general picture

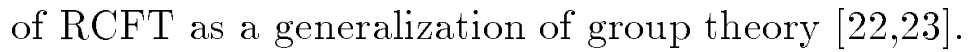

\section{Remarks:}

1. The derivation is valid to leading order in string perturbation theory, which makes it an exact statement for BPS branes. It would be interesting to see if there is a sense in which it is true beyond leading order. Because the result seems very natural to us, we conjecture that it will continue to hold even for nonrational backgrounds.

2. Note that $(2 . \overline{2})$ behaves nicely upon inclusion of Chan-Paton spaces

$$
\sqrt{\operatorname{dim}\left(\mathcal{H}_{\alpha \alpha} \otimes \operatorname{Mat}_{N}(\mathbb{R})\right)}=N \sqrt{\operatorname{dim}\left(\mathcal{H}_{\alpha \alpha}\right)}
$$

Actually, in string theory we impose a reality condition on Chan-Paton factors in $\operatorname{Mat}_{N}(\mathbb{C})$ so that Chan-Paton factors take values in the Hermitian matrices, $H_{N}$, but the real dimension is again $\operatorname{dim} H_{N}=N^{2}$.

3. As mentioned earlier, the quantity $\frac{\psi_{\alpha}{ }^{1}}{\sqrt{S_{11}}}$ is what Affleck and Ludwig call the "nonintegral groundstate degeneracy" in boundary CFT. It has been conjectured, and established in conformal perturbation theory, that this quantity descreases along renormalization group flows. See [1]

\subsection{Example: Compactification on Tori}

A simple example of the above rule is provided by the Gaussian model on $T^{d}$ with constant background metric $G_{\mu \nu}$ and two-form $B_{\mu \nu}$. We set $E=G+B$. (If we pick rational values for the Narain moduli, this case fits simply into the framework discussed above; in the general quasirational case we will take some shortcuts below in showing that the squared tension is given by a regularized dimension, given boundary states satisfying the Cardy condition.) The closed conformal field theory is characterized by a Narain lattice $\Gamma(E) \subset \mathbb{R}^{d, d}$. The isomorphism of $u(1)^{d}$ left and right chiral algebras is given by $\alpha_{n}=\mathcal{R} \cdot \tilde{\alpha}_{-n}$, where $\mathcal{R} \in O(d ; \mathbb{R})$ is some rotation matrix $\left[\begin{array}{ll}1 \\ 1\end{array}, 1\right]$

Let

$$
\Lambda=\Gamma(E) \cap\left\{\left(p_{L} ; p_{R}\right): p_{L}=\mathcal{R} \cdot p_{R}\right\}
$$

We denote the rank of $\Lambda$ by $r$ and the metric tensor on $\Lambda$ by $\mathcal{G}_{i j}$. By this we mean the metric appearing in the (Euclidean) inner product $p_{L}^{2}+p_{R}^{2}=n^{T} \mathcal{G} n$ for a $d$ dimensional vector of integers $n$. Note that $\Lambda$ depends on both $E$ and $\mathcal{R}$. 
Let $\theta$ be a character of $\Lambda$. Cardy states will be of the form

$$
|\theta ; E, \mathcal{R}\rangle\rangle=N_{\theta} \sum_{\lambda \in \Lambda} e^{2 \pi i \theta \cdot \lambda} e^{-S(\mathcal{R})}|\lambda\rangle
$$

where $S(\mathcal{R})=\sum_{n} \alpha_{-n} \mathcal{R} \tilde{\alpha}_{-n} / n$ and we use the discrete measure for the momentum eigenvectors. Let us ask that the single state $|\theta ; E, \mathcal{R}\rangle\rangle$ form a Cardy state. We compute

$$
\left\langle\left\langle\theta\left|q_{c}^{\frac{1}{2}\left(L_{0}+\tilde{L}_{0}-c / 12\right)}\right| \theta\right\rangle\right\rangle=\frac{\left|N_{\theta}\right|^{2}}{\eta\left(q_{c}\right)^{d}} \sum_{\lambda \in \Lambda} q_{c}^{\frac{1}{2} \lambda_{L}^{2}}=\frac{\left|N_{\theta}\right|^{2}}{\eta\left(q_{c}\right)^{d}} \sum_{n \in \mathbb{Z}^{r}} e^{-\pi t_{c} n^{i} \mathcal{G}_{i j} n^{j}}
$$

From Poisson resummation we get

$$
\left\langle\left\langle\theta\left|q_{c}^{\frac{1}{2}\left(L_{0}+\tilde{L}_{0}-c / 12\right)}\right| \theta\right\rangle\right\rangle=t_{c}^{d / 2-r / 2} \frac{\left|N_{\theta}\right|^{2}}{\sqrt{\operatorname{det} \mathcal{G}_{i j}}} \frac{1}{\eta\left(q_{o}\right)^{d}} \sum_{\hat{n} \in \mathbb{Z}^{r}} e^{-\pi t_{o} \hat{n}^{i}\left(\mathcal{G}^{-1}\right)^{i j} \hat{n}^{j}}
$$

and we thus conclude that $r=\operatorname{rank}(\Lambda)=d$. We interpret this to mean that D-branes wrapped on foliating subtori of $T^{d}$ cannot form Cardy states. Moreover, the minimal normalization is $N_{\theta}=\left(\operatorname{det} \mathcal{G}_{i j}\right)^{1 / 4}$ and therefore, the overlap with the unit $u(1)^{d}$ character is $N_{\theta}=\left(\operatorname{det} \mathcal{G}_{i j}\right)^{1 / 4}$. Equivalently, picking the tension out of the leading piece as $q_{c} \rightarrow 0$ in the closed string channel,

$$
\operatorname{dim} \mathcal{H}_{\theta \theta}=\left(\operatorname{det} \mathcal{G}_{i j}\right)^{1 / 2}
$$

For example, choosing the diagonal torus with Narain lattice $\Gamma(E)=\left\{\frac{1}{\sqrt{2}}\left(\frac{n_{i}}{R_{i}}-\right.\right.$ $\left.\left.m_{i} R_{i} ; \frac{n_{i}}{R_{i}}+m_{i} R_{i}\right)\right\}$ and rotation $\mathcal{R}=\operatorname{diag}\left\{-1^{p} ;+1^{d-p}\right\}$ corresponding to a wrapped $p$ brane then one easily finds:

$$
\operatorname{dim} \mathcal{H}_{\theta \theta}=\prod_{i=1}^{p} R_{i} \prod_{i=p+1}^{d} \frac{1}{R_{i}}
$$

If one adds a flat $B$-field and considers a $d$-brane wrapping $T^{d}$ then $\mathcal{R}=E^{-1} E^{t r}$. In this case one finds

$$
\operatorname{dim} \mathcal{H}_{\theta \theta}=\left(\operatorname{det} \mathcal{G}_{i j}\right)^{1 / 2}=\left|\operatorname{det}\left(G_{\mu \nu}+B_{\mu \nu}\right)\right|\left(\operatorname{det} G_{\mu \nu}\right)^{-1 / 2} .
$$

Both of these agree with standard formulae for the mass of a wrapped brane when

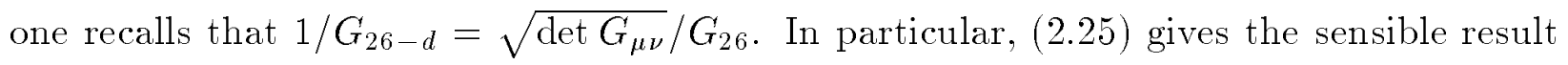
that the tension is given by the Born-Infeld action $\sqrt{\left|\operatorname{det}\left(G_{\mu \nu}+B_{\mu \nu}\right)\right|}$, a result which is essentially already to be found in [i]1]. 


\section{The general argument: Superstrings}

\subsection{Cardy condition for $\mathcal{N}=1$ superconformal field theory}

To define the superconformal algebra we need to pick a spin structure. The index set $\mathcal{I}$ of characters divides into NS and $\mathrm{R}$ sectors which we denote as: $\mathcal{I}_{+}:=\mathcal{I}_{N S}, \mathcal{I}_{-}:=\mathcal{I}_{R}$. Similarly, we must consider both characters and characters twisted by $(-1)^{F}$ :

$$
\chi_{i}^{\epsilon}(q):=\operatorname{Tr}_{\mathcal{H}_{i}}\left(\epsilon^{F} q^{H}\right)
$$

where $\epsilon= \pm 1$. The transformation $\tau \rightarrow-1 / \tau$ acts in a standard way on the spin structures, so we can define several modular matrices:

$$
\begin{aligned}
\chi_{i}^{+}\left(q_{c}\right) & =\sum_{j \in \mathcal{I}_{+}} S_{i j}^{++} \chi_{j}^{+}\left(q_{o}\right) & & i \in \mathcal{I}_{+} \\
\chi_{i}^{-}\left(q_{c}\right) & =\sum_{j \in \mathcal{I}_{-}} S_{i j}^{+-} \chi_{j}^{+}\left(q_{o}\right) & & i \in \mathcal{I}_{+} \\
\chi_{i}^{+}\left(q_{c}\right) & =\sum_{j \in \mathcal{I}_{+}} S_{i j}^{-+} \chi_{j}^{-}\left(q_{o}\right) & & i \in \mathcal{I}_{-} \\
\chi_{i}^{-}\left(q_{c}\right) & =\sum_{j \in \mathcal{I}_{-}} S_{i j}^{--} \chi_{j}^{-}\left(q_{o}\right) & & i \in \mathcal{I}_{-}
\end{aligned}
$$

Note that $\left(S^{++}\right)^{2}=C$ is the conjugation matrix (and for simplicity we will now assume reps are self-conjugate). $S_{i j}^{--}=\delta_{i j}$ because the Witten index is modular invariant. $\overline{\beta_{1}^{\prime}}$

We can form character states $|i ; \eta\rangle\rangle$ in the standard way. $\eta$ denotes the choice of isomorphism between left and right supercurrents so the character basis gives a basis of solutions to the linear equations

$$
\left.\left(G_{r}-i \eta \tilde{G}_{-r}\right)|\alpha\rangle\right\rangle=0
$$

where $r \in \mathbb{Z}$ in the $\mathrm{R}$ sector and $r \in \mathbb{Z}+1 / 2$ in the NS sector.

We can form Cardy states in the usual way:

$$
\left.|\alpha, \epsilon ; \eta\rangle\rangle=\sum_{i \in \mathcal{I}_{\epsilon}} \frac{\psi_{\alpha}^{i}(\eta)}{\sqrt{S_{i 1}^{\epsilon,+}}}|i ; \eta\rangle\right\rangle
$$

3 Warning: We are using a possibly confusing piece of notation. $\chi_{i}^{+}$for $i \in \mathcal{I}_{+}$is the path integral for the spin structure commonly denoted $(-,-)$ ! 
The Cardy condition becomes

$$
\left\langle\left\langle\alpha, \epsilon ; \eta\left|q_{c}^{\frac{1}{2}\left(L_{0}+\tilde{L}_{0}-\frac{c}{12}\right)}\right| \beta, \epsilon ; \eta^{\prime}\right\rangle\right\rangle=\operatorname{Tr}_{\mathcal{H}_{\alpha \beta}^{\eta \eta^{\prime}}} \epsilon^{F} q_{o}^{H_{o}}=\sum_{j \in \mathcal{I}_{\eta \eta^{\prime}}}\left(n_{j}^{\epsilon, \eta \eta^{\prime}}\right)_{\beta}{ }^{\alpha} \chi_{j}^{\epsilon}\left(q_{o}\right)
$$

In a standard unitary theory we will require that $\left(n_{j}^{\epsilon, \eta \eta^{\prime}}\right)_{\alpha}^{\beta} \geq 0$.

In particular, focusing on the $(-,-)$ spin structure, which is invariant under $\tau \rightarrow$ $-1 / \tau$, we have:

$$
\left(n_{i}^{++}\right)_{\alpha}^{\beta}=\sum_{j \in \mathcal{I}_{+}} \frac{S_{i j}^{++}}{S_{1 j}^{++}} \psi_{\alpha}^{j}(\eta)\left(\psi_{\beta}^{j}(\eta)\right)^{*}
$$

We find again that

$$
\operatorname{dim} \mathcal{H}_{\alpha \alpha}^{N S}=\operatorname{dim} \mathcal{H}_{\alpha, \alpha}^{\eta, \eta}=\left(\frac{\psi_{\alpha}{ }^{1}(\eta)}{\sqrt{S_{11}^{++}}}\right)^{2}=\sum_{j \in \mathcal{I}_{+}}\left(n_{j}^{++}\right)_{\alpha}{ }^{\alpha} S_{j 1}^{++}
$$

\section{Remarks:}

1. Equation ( $\left(\overline{3}_{1}^{\prime}\right)$ is only one of several choices one must make in the isomorphism of left and right chiral algebras.

2. In $\left(3.4_{1}^{4}\right)$ we are considering a Cardy set with states with fixed $\eta$ and which are either purely NS or purely R. To make GSO invariant states it is necessary to combine states with different $\eta$ as described below. We can also form Cardy sets with states which are linear combinations of character states from the $R$ and NS sector, and this is required in order to obtain BPS boundary states. There are only minor modifications to the analysis.

3. In $(3 . \overline{3}) \psi_{\alpha}^{1}(\eta)$ is independent of $\eta$.

4. In $\left(\hat{L}_{i}^{-} \bar{i}_{1}\right) n_{j}^{++} \geq 0$, and if the unit operator is present then $n_{j}^{++}$is positive definite for $j=0$. Thus, as for the bosonic string, we can conclude that the right hand side is positive.

\subsection{Tension and dimension for the superstring}

We begin with a product of superconformal theories $\mathcal{C}_{1}\left(\mathbb{R}^{8-d}\right) \otimes \mathcal{C}_{2}$ where $\mathcal{C}_{1}\left(\mathbb{R}^{8-d}\right)$ is the lightcone superconformal field theory of $8-d$ free bosons and fermions $X^{\mu}, \psi^{\mu} . \mathcal{C}_{2}$ is a unitary superconformal field theory with $\hat{c}=\hat{\bar{c}}=d$. The closed string is a subtheory 
because (a) we restrict to the subspace with the same spin structures on both factors and (b) we GSO project. The statespace has the form:

$$
\left(\mathcal{H}_{N S}^{1} \otimes \mathcal{H}_{N S}^{2}\right)^{+} \oplus\left(\mathcal{H}_{R}^{1} \otimes \mathcal{H}_{R}^{2}\right)^{+}
$$

where the + superscript indicates the need to take a GSO projection. We will take the brane to be a point particle in the uncompactified dimensions. The general form of the boundary state is then [i] $[1]$

$$
\begin{gathered}
\left.\left.\left.\left.C^{\alpha, N S,+}|x, N S ; \eta\rangle\right\rangle^{(1)} \otimes|\alpha, N S ; \eta\rangle\right\rangle^{(2)}+C^{\alpha, N S,-}|x, N S ;-\eta\rangle\right\rangle^{(1)} \otimes|\alpha, N S ;-\eta\rangle\right\rangle^{(2)} \\
\left.\left.\left.\left.+C^{\alpha, R,+}|x, R ; \eta\rangle\right\rangle^{(1)} \otimes|\alpha, R ; \eta\rangle\right\rangle^{(2)}+C^{\alpha, R,-}|x, R ;-\eta\rangle\right\rangle^{(1)} \otimes|\alpha, R ;-\eta\rangle\right\rangle^{(2)}
\end{gathered}
$$

Here we will assume the state is made from a Cardy set of boundary states of the internal theory, although this is probably not necessary (i.e., only the combined theory really needs to obey sewing axioms). The constants are determined by imposing the GSO projection, by choosing BPS or anti-BPS branes, and by Cardy's condition. In supersymmetric type II theory the GSO projection requires $C^{\alpha, N S,-}=-C^{\alpha, N S,+}$ and $C^{\alpha, R,+}=C^{\alpha, R,-}$. Note that the GSO projection does not project out the unit operator in the internal theory. Other choices of GSO projection will lead to other conditions on the coefficients. For example, in type 0 theory where one has a diagonal GSO projection, $C^{\alpha, N S,+}$ and $C^{\alpha, N S,-}$ are independent, reflecting the doubling of the number of D-brane states [2]

The graviton vertex operator is $\epsilon_{\mu \nu} \psi^{\mu} \tilde{\psi}^{\nu} e^{i k X} \otimes 1$ so the coupling of the state ( $\left.\overline{3}_{0} . \bar{g}_{1}^{\prime}\right)$ to the graviton is, up to a phase,

$$
\left(C^{\alpha, N S,+}-C^{\alpha, N S,-}\right) \frac{\psi_{\alpha}^{1}(\eta)}{\sqrt{S_{11}^{++}}}
$$

We can obtain a quantization condition on the coefficient $C^{N S}$ in order to ensure that the combined state continues to satisfy the Cardy condition. The condition depends on whether or not we want to enforce the GSO projection in the open string channel (i.e. whether or not we consider a BPS or a non-BPS type brane). If we do not enforce the condition then $C^{R}=0$ and $2\left|C^{N S}\right|^{2} \in \mathbb{Z}_{+}$. Taking the minimal value allowed we get the open string NS sector is

$$
\operatorname{Tr}_{\mathcal{H}_{\alpha \alpha}^{N S}} q_{o}^{H_{o}}=\frac{1}{\eta^{(8-d) / 2}}\left(\frac{\vartheta_{3}}{\eta}\right)^{(8-d) / 2} \sum_{i \in \mathcal{I}_{+}} n_{\alpha \alpha}^{i} \chi_{i}^{+}\left(q_{o}\right)
$$


If we want to have the usual GSO projection then we take a bilinear form for the cylinder amplitude $\stackrel{i=1}{-1}$ and impose $2\left(C_{12}^{N S}\right)^{2}=-\left(C_{12}^{R}\right)^{2} / 8=\frac{n}{2}$ and taking the minimal value $n=1$ we have

$$
\operatorname{Tr}_{\mathcal{H}_{\alpha \alpha}^{N S}} q_{o}^{H_{o}}=\frac{1}{\eta^{(8-d) / 2}} \frac{1}{2}\left[\left(\frac{\vartheta_{3}}{\eta}\right)^{(8-d) / 2} \sum_{i \in \mathcal{I}_{+}} n_{\alpha \alpha}^{i} \chi_{i}^{+}\left(q_{o}\right)+\left(\frac{\vartheta_{4}}{\eta}\right)^{(8-d) / 2} \sum_{i \in \mathcal{I}_{+}} n_{\alpha \alpha}^{i} \chi_{i}^{-}\left(q_{o}\right)\right]
$$

In either case, taking into account $\left(3_{3} \overline{1} \overline{0}_{1}^{\prime}\right)$ and fixing the overall normalization from the torus case we get the superanalog of $\left(2_{2}^{2} .3^{1}\right)$ :

$$
\left(\ell_{s} M\right)^{2}=\frac{1}{16 \pi^{2}} \frac{\left(2 \pi \ell_{s}\right)^{8-d}}{G_{10-d}} \operatorname{dim} \mathcal{H}_{\alpha \alpha}^{N S}
$$

Here $\mathcal{H}_{\alpha \alpha}^{N S}$ is the open string channel NS sector of the full string theory. In particular, this result holds for BPS and nonBPS, the difference of a factor of $\sqrt{2}$ in the tension between a BPS and non-BPS brane [2-6] is due to the factor of $1 / 2$ in the projection operator which reduces the dimension of $\mathcal{H}_{\alpha \alpha}^{N S}$ by a factor of two.

\section{Discussion}

We conclude with two speculative remarks on possible future applications of this work.

First, it is natural to speculate that the mass formula derived here in terms of the Affleck-Ludwig degeneracy $g$

$$
\mathcal{M}^{2} \sim|g|^{2}=\operatorname{Tr}_{\mathcal{H}_{\alpha \alpha}}(1)
$$

could play a role in extensions of the attractor mechanism [2] to nonsupersymmetric, spherically symmetric charged black holes. In the context of BPS black holes in $\mathcal{N}=2$ supersymmetric compactifications to four dimensions, it is clear that $g$ can be identified with the central charge $|\mathcal{Z}|$. The attractor mechanism tells us that we should associate spherically symmetric extremal RR charged black holes with gradient flow on the moduli

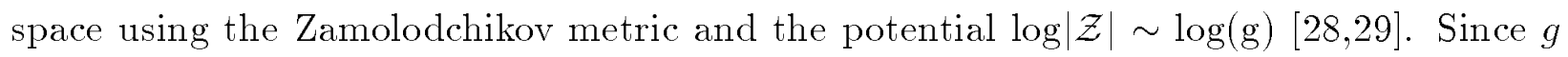
is intrinsically well defined in string theory without any reference to supersymmetry, one can conjecture that in general compactifications, one can associate spherically symmetric extremal RR charged black hole solutions with gradient flows using the Zamolodchikov

4 Thus changing the orientation so that we have two ingoing circles. This is necesary to get the spacetime fermion minus sign. 
metric and the potential $\log (g)$. The "doubly extremal" solutions (with constant values of the closed string moduli as a function of the radius) then arise when one chooses the moduli to sit at a (local) minimum of $g$. It would also be interesting to see if the dynamical evolution of couplings on a test 3-brane falling into a black hole is related to renormalization group flow of the boundary entropy.

Second, our result fits in well with the currently emerging interconnections between D-branes, K-theory, and noncommutative geometry. In the framework of noncommutative

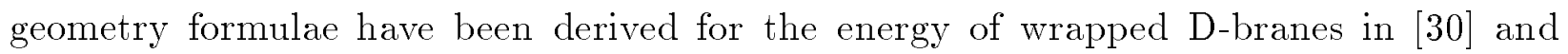
subsequent papers. See [3]1] for a recent discussion. In order to account for the mass $\mathcal{M}$ of a wrapped D-brane in its groundstate one must add to the noncommutative Yang-Mills action a term proportional to $\operatorname{Tr}_{E}(1)$, where $E$ is the projective module of sections of the (noncommutative) Chan-Paton bundle. On the other hand, in the present paper we have derived a relation of the form $\mathcal{M}^{2} \sim \operatorname{Tr}_{\mathcal{H}_{\alpha \alpha}}(1)$. The two results are compatible if we can identify (at least in the $\alpha^{\prime} \rightarrow 0$ limit corresponding to the NCSYM theory) $\mathcal{H}_{\alpha \alpha}=\operatorname{End}(E)$, and such an identification in this limit is strongly suggested by the behavior of the finite dimensional Chan-Paton factors. Clearly, this connection deserves closer scrutiny.

It is also worth noting that in the theory of operator algebras one can define the Murray-von Neumann dimension, spaces of operators. It is well-known that, at least in some RCFTs, the regularized dimensions (2:1 1 in $)$ are indeed given by ratios of such Murray-von Neumann dimensions. In this way the Jones index of subfactors appears in RCFT. On the other hand, these same dimensions can be related to traces of projection operators on towers of finite-dimensional

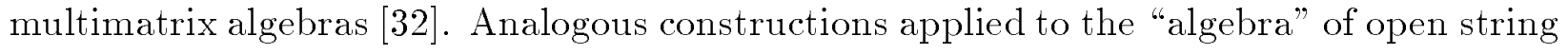
vertex operators might lead to interesting new ways of formulating D-branes, and, perhaps, even $M$-theory.

\section{Acknowledgements}

We would like to thank T. Banks, J. Cardy, M. Douglas, P. Fendley, D. Friedan, J. Maldacena, A. Recknagel, H. Saleur, V. Schomerus, N. Seiberg, I. Singer and E. Witten for useful discussions and remarks. We would like to acknowledge the hospitality of the Aspen Center for Physics and the Amsterdam Summer Workshop on String Theory. GM would like to thank the Institute for Advanced Study for hospitality and the Monell foundation for

5 In the literature on operator algebras this is also called the "coupling constant." 
support. The work of JH is supported by NSF Grant No. PHY 9901194, SK is supported by an A.P. Sloan Foundation Fellowship and a DOE OJI Award, GM is supported by DOE grant DE-FG02-92ER40704, and ES is supported by an A.P. Sloan Foundation Fellowship, a DOE OJI Award, and by the DOE under contract DE-AC03-76SF00515. 


\section{References}

[1] C. G. Callan, C. Lovelace, C. R. Nappi and S. A. Yost, "Adding Holes and Corsscaps to the Superstring," Nucl. Phys. B293 (1987) 83; C.G. Callan, C. Lovelace, C. R. Nappi and S. A. Yost, "Loop Corrections to Superstring Equations of Motion," Nucl. Phys. B308 (1988) 221.

[2] J. Polchinski and Y. Cai, "Consistency of Open Superstring Theories," Nucl. Phys. B296 (1988) 91.

[3] J. Cardy, "Boundary conditions, fusion rules, and the Verlinde formula," Nucl. Phys. B324 (1989) 581.

[4] D. Lewellen, "Sewing constraints for conformal field theories on surfaces with boundaries," Nucl.Phys. B372 (1992) 654; J.L. Cardy and D.C. Lewellen, "Bulk and boundary operators in conformal field theory," Phys.Lett. B259 (1991) 274.

[5] D. Friedan, "The space of conformal field theories for the $c=1$ Gaussian model," unpublished, c. 1994.

[6] G. Pradisi, A. Sagnotti and Ya. S. Stanev, "Planar Duality in SU(2) WZW Models,"

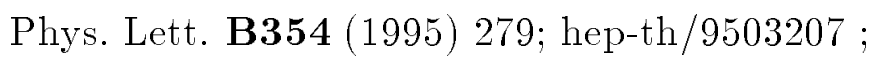

G. Pradisi A. Sagnotti and Ya. S. Stanev, "The Open Descendants of Nondiagonal

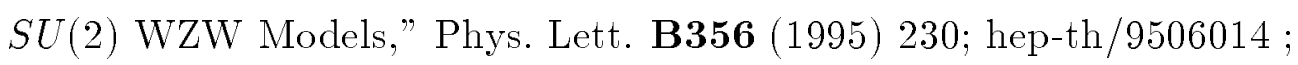

G. Pradisi, A. Sagnotii, and Ya. S. Stanev, "Completeness conditions for boundary

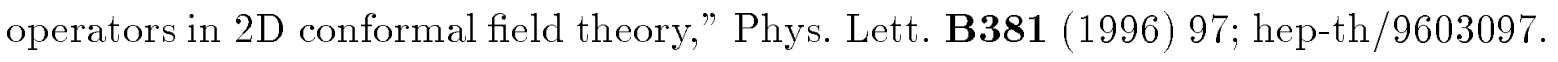

[7] M. Li, Nucl. Phys. B460 (1996) 351;

[8] P. Di Vecchia, M. Frau, I. Pesando, S. Sciuto, A. Lerda, and R. Russo, "Classical p-branes from boundary state," hepepth $\angle \overline{9} \overline{0} \overline{0} \overline{0} \overline{0} \overline{6} \overline{8}$.

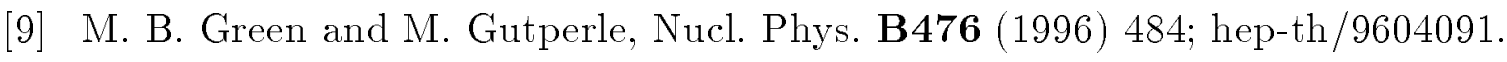

[10] O. Bergman and M. Gaberdiel, "A Non-supersymmetric Open String Theory and

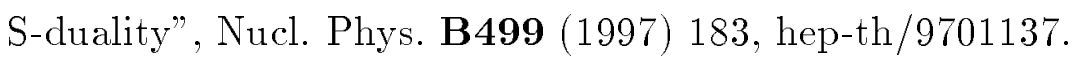

[11] J. Fuchs and C. Schweigert, "A classifying algebra for boundary conditions," "hepe-

J. Fuchs and C. Schweigert, "Branes: from free fields to general backgrounds," Nucl.

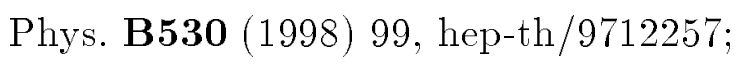

J. Fuchs and C. Schweigert, "Symmetry breaking boundaries I. General theory," "hesp=:

J. Fuchs and C. Schweigert, "Symmetry Breaking Boundaries. 2. More Struc-

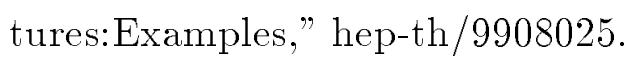

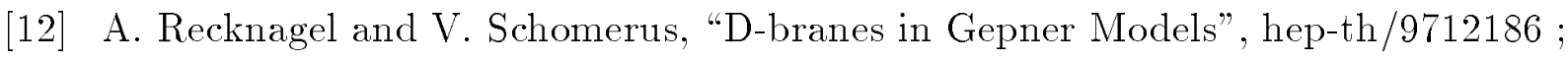

A. Recknagel and V. Schomerus, "Boundary Deformation Theory and Moduli Spaces

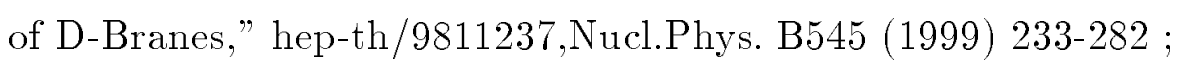


A. Recknagel and V. Schomerus, "Moduli Spaces of D-branes in CFT-backgrounds,"

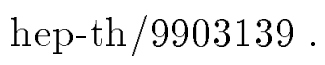

[13] I. Brunner, M. Douglas, A. Lawrence, C. Romelsberger, "D-branes on the Quintic,"

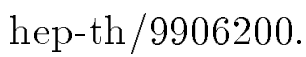

[14] R.E. Behrend, P.A. Pearce, V.B. Petkova, J-B Zuber, "Boundary conditions in Rational Conformal Field Theories," hiep-th

[15] A. Cohen, G. Moore, P. Nelson, and J. Polchinski, "An invariant string propagator," in Unified String Theories, p. 568, M. Green and D. Gross, eds. World Scientific, 1986.

[16] N. Ishibashi, "The Boundary and Crosscap States in Conformal Field Theories", Mod. Phys. Lett. A4 (1989) 251; N. Ishibashi and T. Onagi, "Conformal Field Theories on Boundaries and Surfaces with Crosscaps", Mod. Phys. Lett. A4 (1989) 161.

[17] I. Affleck and A.W.W. Ludwig, "Universal noninteger ground state degeneracy in critical quantum systems," Phys.Rev.Lett.67 (1991) 161.

[18] C. G. Callan, Jr. and I. R. Klebanov, "D-Brane Boundary State Dynamics," Nucl.

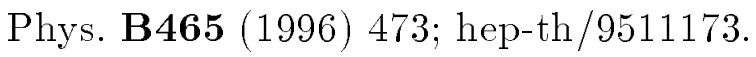

[19] S. Elitzur, E. Rabinovici and G. Sarkissian, "On Least Action D-Branes," Nucl. Phys. B541 (1999) 731 ; hè

[20] J. Polchinski, String Theory, Cambridge Univ. Press, 1998

[21] R. Dijkgraaf and E. Verlinde, "Modular invariance and the fusion algebra," Presented at Annecy Conf. on Conformal Field Theory, Annecy, France, Mar 14-16, 1988. Published in Annecy Field Theory 1988:0087

[22] G. Moore and N. Seiberg, "Naturality in conformal field theory," Nuc. Phys. B313 (1989) 16

[23] G. Moore and N. Seiberg, "Classical and Quantum Conformal Field Theory," Commun. Math. Phys. 123(1989)177; "Lectures on Rational Conformal Field Theory," in Strings '89,Proceedings of the Trieste Spring School on Superstrings, 3-14 April 1989, M. Green, et. al. Eds. World Scientific, 1990

[24] I. Affleck and A. W. W. Ludwig, Phys. Rev. B48 (1993) 7297.

[25] M. Billo, B. Craps and F. Roose, "On D-Branes in Type 0 String Theory," Phys. Lett. B457 (1999) 61; hè

[26] A. Sen, "NonBPS States and Branes in String Theory," hiep-thh

[27] S. Ferrara, R. Kallosh and A. Strominger, "N=2 Extremal Black Holes," Phys. Rev. D52 (1995) 5412,

[28] S. Ferrara, G. W. Gibbons, R. Kallosh, "Black Holes and Critical Points in Moduli

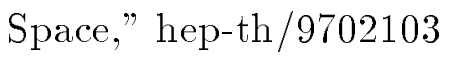

[29] G. Moore, "Arithmetic and attractors," hiep

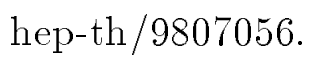

[30] A. Connes, M.R. Douglas, and A. Schwarz, "Noncommutative geometry and Matrix Theory: Compactification on Tori," hep-th/9 
[31] B. Pioline and A. Schwarz, "Morita equivalence and $T$-duality (or $B$ versus $\Theta$ )," "hepi (1)

[32] F.M. Goodman, P. de la Harpe, and V.F.R. Jones, Coxeter Graphs and Towers of Algebras, MSRI publications 14, Springer-Verlag 1989. 